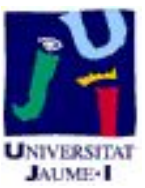

Título artículo / Títol article: Apparel sizing using trimmed PAM and OWA operators

Autores / Autors

Ibáñez Gual, María Victoria ; Epifanio López, Irene ; Simó Vidal, Amelia ; Vinué Visús, Guillermo ; Alemany Mut, Sandra ; Domingo Esteve, Juan de Mata ; Ayala Gallego, Guillermo

Revista:

Expert Systems with Applications, v.39, n. 12 (September 2012)

Versión / Versió:

Preprint

Cita bibliográfica / Cita bibliogràfica (ISO 690):
IBÁÑEZ, M. Victoria, et al. Apparel sizing using trimmed PAM and OWA operators. Expert Systems with Applications, 2012, vol. 39, no 12, p. 1051210520.

url Repositori UJI: $\quad$ http://hdl.handle.net/10234/70403 


\title{
Apparel sizing using trimmed PAM and OWA operators
}

\author{
M. V. Ibáñez ${ }^{(1)}$, G. Vinué( ${ }^{(2)}$, S. Alemany ${ }^{(3)}$, A. Simó( ${ }^{(1)}$, I. Epifanio $^{(1)}$, J. \\ Domingo $^{(4)}$, G. Ayala ${ }^{(2)}$ \\ (1).Department of Mathematics. University Jaume I. Castellón. Spain. (2) Department \\ of Statistics and O.R., University of Valencia, Valencia, Spain. (3) Biomechanics \\ Institute of Valencia, Universidad Politécnica de Valencia, Valencia, Spain, (4) Dept. of \\ Informatics, University of Valencia, Valencia, Spain
}

\begin{abstract}
This paper is concerned with apparel sizing system design. One of the most important issues in the apparel development process is to define a sizing system that provides a good fit to the majority of the population. A sizing system classifies a specific population into homogeneous subgroups based on some key body dimensions. Standard sizing systems range linearly from very small to very large. However, anthropometric measures do not grow linearly with size, so they can not accommodate all body types. It is important to determine each class in the sizing system based on a real prototype that is as representative as possible of each class. In this paper we propose a methodology to develop an efficient apparel sizing system based on clustering techniques jointly with OWA operators. Our approach is a natural extension and improvement of the methodology proposed by McCulloch et al in 1998 [22], and we apply it to the anthropometric database obtained from a anthropometric survey of the Spanish female population, performed during 2006.
\end{abstract}

Keywords: Anthropometric data, Sizing systems, Trimmed k-medoids, OWA operators.

Email address: mibanez@mat.uji.es, guillermo.vinue@uv.es, sandra.alemany@ibv.upv.es, simo@mat.uji.es, epifanio@mat.uji.es, juan.domingo@uv.es, guillermo.ayala@uv.es (M. V. Ibáñez ${ }^{(1)}$, G. Vinué( ${ }^{(2)}$, S.

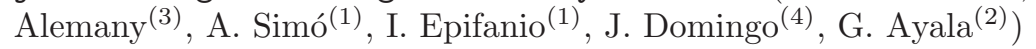




\section{Introduction}

The development of Ready To Wear (RTW) cloth requires an estimation of body measures of the target population to generate sizing charts, patterns on a basic size and grading parameters. However, most apparel manufacturers create and adjust their own size charts by trial and error using small customer surveys, mainly models representing the basic size, plus analysis of sales and returned merchandising reports [5]. The growing relocation of the pattern and production activities and the poor level of application of sizing standards also produce that one of the main clothing complaints is the lack of fitting.

There are several local and international standards proposing a regulation of the sizing system based on key anthropometric measures, but the lack of common rules and criteria is one of the drawbacks for their implementation. In this context, 'vanity sizing' grows as a common practice among clothing companies. With this strategy, companies often adjust the measurement specifications for each size based on a sale strategy designed to make consumers, especially women, feel better about fitting into smaller sizes [8, 1], and therefore prompting them to buy more. This system contributes to difficult customers to find the correct size in different companies. In fact, nowadays, the correct size selection is the main obstacle to large scale online garment sales because it is difficult to find the fit garment from the general size information.

A sizing system classifies a specific population into homogeneous subgroups based on some key body dimensions [6]. The major dilemma is to decide into how many size groups should the population be divided, in order to optimize benefits and user satisfaction. Most of the standard sizing charts propose sizes based on intervals over just one anthropometric dimension. Current standards consider the low correlation between some key dimensions and use bivariate distributions to define a sizing chart and cross tabulation to select the sizes covering the highest percentage of population. For lower limb garments European standards [7] propose the combination of three anthropometric dimensions (waist girth, hip girth and stature) leading to a significant increase of the number of sizes which are low profitable for the companies since there are very far from the current offer. Moreover, correlations between anthropometric measures show a great variability on body proportion. It is not possible to cover these different body morphologies with these kind of models. That is why, multivariate approaches 
have been proposed to develop sizing systems. Principal components are often used to reduce the dimension of our anthropometric data set, and the two first principal components are used to generate bivariate distributions $[4,13,20,12,14,27]$. As an alternative to bivariate distributions, clustering techniques using partitioning methods, like k-means algorithms, group the population into morphologies using the complete set of anthropometric variables as input $[6,32,23])$. A large scale implementation of this statistical approach using data mining and decision trees was proposed in [15] and [2]. Different alternative approaches, based on optimization algorithms, were first proposed by Tryfos [30], who used integer programming to partition the body dimension space into a discrete set of sizes by choosing the size system to optimize the sales of garment. Later on, McCulloch et al. [22] modified this approach by focusing the problem on the quality of fit instead on of the sales. The sizes were determined by means of a nonlinear optimization problem. The objective function measured the misfit between a person and the prototype, using a particular dissimilarity measure and removing from the data set a prefixed proportion of the sample. In this paper, we are going to follow with this idea. In fact, our paper has been conceived as an extension of the work of McCulloch et al. [22].

All these multivariate approaches based on optimization algorithms, need the previous definition of an objective function. These functions basically measure the misfit between a feature vector from a given person and a model or prototype by combining the misfit observed for each feature. It is clear that discrepancies in certain features (or dimensions) are more critical than others. It is important to get a meaningful combination of these discrepancies. In this sense, the Analytic Hierarchy Process (AHP) proposed in [26], tries to convert subjective assessments of relative importance into a set of overall scores or weights. AHP is one of the more widely applied multi-attribute decision making methods. Applied to the customized garment design process, Chen et al. [5] propose ordered weighted averaging operators (OWA) jointly with fuzzy methods, to model the easy allowance of the 2D patterns. The weights of the OWA operators can be used to adjust the compromise between the style of garments and the general comfort sensation of wearers.

Fitting RTW clothes is a problem for both customer and apparel industry [8]. For this reason during last years both national administrations and industrial groups of the clothing sector have been fostering national anthropometric surveys in different countries: USA, UK, France, Australia, Spain and Germany among others. These studies show that there is a high percent- 
age of population with difficulties to find proper fit cloth. Anthropometric studies carried out up to date show high percentages of population with fitting problems. Studies carried out in UK [29] and Germany [6], show a $60 \%$ and $50 \%$ respectively of customers with difficulty to find proper clothes. In the same way, an anthropometric study performed in USA [9] to update the sizing ASTM standards also concluded that a $54 \%$ of the population was not satisfied with the fitting of the ready to wear (RTW) cloth [3]. Additionally, from the technological point of view, new 3D body scanning techniques constitute a step forward in the way of conducting and analyzing anthropometric data and contribute to promote new anthropometric surveys. As a result, broad anthropometric databases are available and constitute valuable information to improve garment fitting adapted to the body shape of the population starting from the definition of an optimized sizing system.

In this way, a national 3D anthropometric survey of the female population was conducted in Spain in 2006 by the Spanish Ministry of Health. The aim of this survey was to generate anthropometric data from the female population addressed to the clothing industry. In this study, a sample of 10.415 Spanish females from 12 to 70 years old randomly selected was measured using a 3D body scanner and 95 anthropometric measures were obtained (Anthropometric survey).

In this paper, we propose a methodology that combines some of these approaches in order to develop a more efficient apparel sizing system that can increase accommodation of the population. We apply it to the anthropometric survey data of the Spanish female population (Anthropometric survey). Our approach is close to that of McCulloch et al. [22]. However, there are two main differences. First, when looking for the $k$ prototypes, we use a trimmed k-medoid clustering method i.e. a trimmed version of the Partitioning Around Medoids (PAM) algorithm, instead of the continuous optimization problem proposed by McCulloch et al. [22]. So, our aim is to look for medoids i.e. for typical persons within the sample, which means that our final prototypes will be real persons of the data set. Additionally, we take into account that an apparel sizing system is intended to cover only what we could call standard population, leaving out those individuals who might be considered outliers respect to a set of measurements. For this reason we propose the use of a trimmed version of PAM procedure. Second, the dissimilarity measure proposed by McCulloch et al. [22], is merely based on the sum of squared discrepancies over each individual feature. We propose to modify this dissimilarity measure by taking into account to the user, using 
an OWA operator.

The outline of the paper is as follows. Section 2 proposes the methodology. The description of our data set is given in Section 3 . The application of our procedure to the anthropometric database of Spanish women is given in Section 4. Conclusions and possible further developments conclude the paper in Section 5 .

\section{Methodology}

When we talk about an apparel sizing system, our target population is not the whole population. An apparel sizing system is intended to cover only that we could call standard population, leaving out those individuals who might be considered as outliers regarding to a set of measurements.

As it has been stated in the introduction, the methodology that we propose is based on two basic ideas: the use of a trimmed version of the $k$ medoids algorithm and the use of OWA operators to combine the individual discrepancies proposed by McCulloch et al. [22]. Our aim in this section is to explain these ideas in a detailed way.

\subsection{Trimmed $k$-medoids}

A classical partitioning cluster method is the well-known $k$-means method. However, the $k$-means method is not a robust procedure, and their results can be influenced by outliers and extreme data, or bridging points between clusters. Trimmed $k$-means is one way of increasing robustness of the $k$ means which combines the $k$-means main idea with a impartial trimming procedure [10] in such a way that a proportion $\alpha$ (between 0 and 1) of observations are trimmed. Trimmed $k$-means is analogous to $k$-means but a proportion $\alpha$ of observations is discarded by the own procedure where the trimmed observations are self-determined by the data.

Let $x_{1}, \ldots, x_{n}$ be $n$ observations of dimension $p$. Let $k$ be the number of groups. The $k$-means method searches for a set of $k$ points, $m_{1}^{*}, \ldots, m_{k}^{*}$, the centroids, verifying

$$
\left\{m_{1}^{*}, \ldots, m_{k}^{*}\right\}=\operatorname{argmin}_{m_{1}, \ldots, m_{k}} \frac{1}{n} \sum_{i=1}^{n} \inf _{1 \leq j \leq k}\left\|x_{i}-m_{j}\right\|^{2},
$$

and each point $x_{i}$ is assigned to its closest center $m_{j}^{*}$. Given $k$ and the 
trimming size $\alpha$, trimmed $k$-means searches $k$ points, $m_{1}^{*}, \ldots, m_{k}^{*}$ such that

$$
\left\{m_{1}^{*}, \ldots, m_{k}^{*}\right\}=\operatorname{argmin}_{\mathbf{Y},\left\{\mathbf{m}_{\mathbf{1}}, \ldots, \mathbf{m}_{\mathbf{k}}\right\}} \frac{1}{\lceil n(1-\alpha)\rceil} \sum_{x_{i} \in \mathbf{Y}} \inf _{1 \leq j \leq k}\left\|x_{i}-m_{j}\right\|^{2},
$$

where $\mathbf{Y}$ ranges on subsets of $x_{1}, \ldots, x_{n}$ containing $\lceil n(1-\alpha)\rceil$ data points, and $\lceil\cdot\rceil$ denotes the integer part of a given value. Each non-trimmed point $x_{i}$ is assigned to its closest centroid $m_{j}$. An algorithm for computing trimmed $k$ - means can be found in [11], and it is available at the $\mathrm{R}$ [25] package tclust [16].

Instead of using trimmed $k$-means, we will use a modified version, the trimmed $k$-medoids, joining the best of the $k$-medoids and trimmed $k$-means. The $k$-medoids algorithm is based on finding $k$ representative subjects (also known as medoids [17]) from the data set in such a way that the sum of the within cluster dissimilarities is minimized, instead of minimizing the squared distances as in $k$-means. Methods based on the minimization of sums (or averages) of dissimilarities (the so-called $L^{1}$ methods) are much more robust to outliers than methods based on sums of squares, such as $k$-means. Note also that the centroids from the $k$-means do not have to be one of the subjects in the original data set. This has been one of our principal motivation for selecting the trimmed $k$-medoid method, because medoids are representative subjects in the clusters, very useful in our application. Another reason was the possibility of applying the $k$-medoid to data described only by dissimilarities. The medoids always exist, even when the data can by related only by a collection of dissimilarities. We just have to compute the dissimilarities between our subjects, there is no need to calculate cluster centers or centroids.

Trimmed $k$-medoids is analogous to $k$-medoids but a proportion $\alpha$ of observations is discarded by the own procedure (the trimmed observations are self-determined by the data as before). Furthermore, trimmed $k$-medoids are analogous to trimmed $k$-means. Let $d\left(x_{i}, x_{j}\right)$ be the dissimilarity between subjects $i$ and $j$. For a given $k$ and the trimming proportion $\alpha$, trimmed $k$ medoids searches $k$ subjects of the data, $x_{i_{1}}^{*}, \ldots, x_{i_{k}}^{*}$ such that

$$
\left\{x_{i_{1}}^{*}, \ldots, x_{i_{k}}^{*}\right\}=\operatorname{argmin}_{\mathbf{Y}, \mathbf{x}_{\mathbf{i}_{1}}, \ldots, \mathbf{x}_{\mathbf{i}_{\mathbf{k}}}} \frac{1}{\lceil n(1-\alpha)\rceil} \sum_{x_{i} \in \mathbf{Y}} \inf _{1 \leq j \leq k} d\left(x_{i}, x_{i_{j}}\right),
$$

where $\mathbf{Y}$ ranges on subsets of $x_{1}, \ldots, x_{n}$ containing $\lceil n(1-\alpha)\rceil$ data points, and $[\cdot]$ denotes the integer part of a given value. Each non-trimmed point 
$x_{i}$ is assigned to its closest medoid $x_{i_{j}}^{*}$. The algorithm of [11] can be easily adapted for computing trimmed $k$-medoids. The detailed algorithm is given in 1 .

Note that the medoid of a group can be computed with function pam (with $k=1$ for each group) from the $\mathrm{R}$ package cluster [21].

In short, we can describe the algorithm as

1. Select $k$ starting points that will serve as seed medoids.

2. Assume that $x_{i_{1}}, \ldots, x_{i_{k}}$ are the $k$ medoids obtained in the previous iteration:

(a) Assign each observation to its nearest medoid:

$$
d_{i}=\min _{j=1, \ldots k} d\left(x_{i}, x_{i_{j}}\right), \quad i=1, \ldots, n
$$

and keep the set $H$ having the $\lceil n(1-\alpha)\rceil$ observations with lowest $d_{i}$ 's.

(b) Split $H$ into $H=\left\{H_{1}, \ldots, H_{k}\right\}$ where the points in $H_{j}$ are those closer to $x_{i_{j}}$ than to any of the other medoids.

(c) The medoid $x_{i_{j}}$ for the next iteration will be the medoid of observations belonging to group $H_{j}$.

3. Repeat the step 2 a few times. After these iterations, compute the final evaluation function.

This algorithm is repeated a few times and the best solution is preserved, see 1 .

Next section comments the dissimilarity used.

\subsection{Dissimilarity measure}

As was said before, the dissimilarity used to quantify the misfit between an individual and the prototype is a key ingredient to obtain an efficient sizing system. Let us start by introducing some notation. Each individual in the data set is represented by a feature vector of size $p$ of their body measurements, $x=\left(x_{1}, \ldots, x_{p}\right)$, and $d_{i}(x, y)$ denotes the dissimilarity in the 


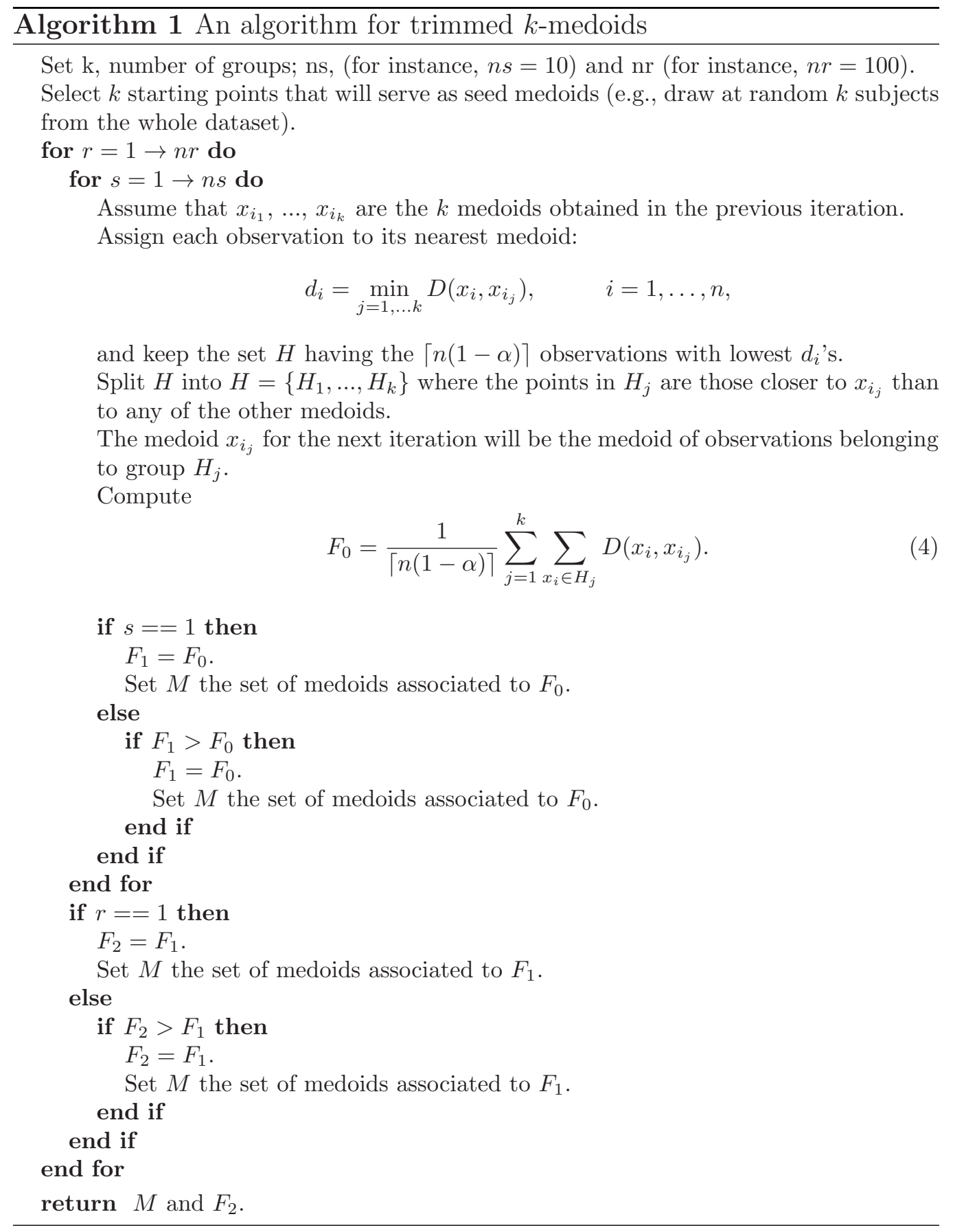


$i$ th feature between individuals $x$ and $y$.

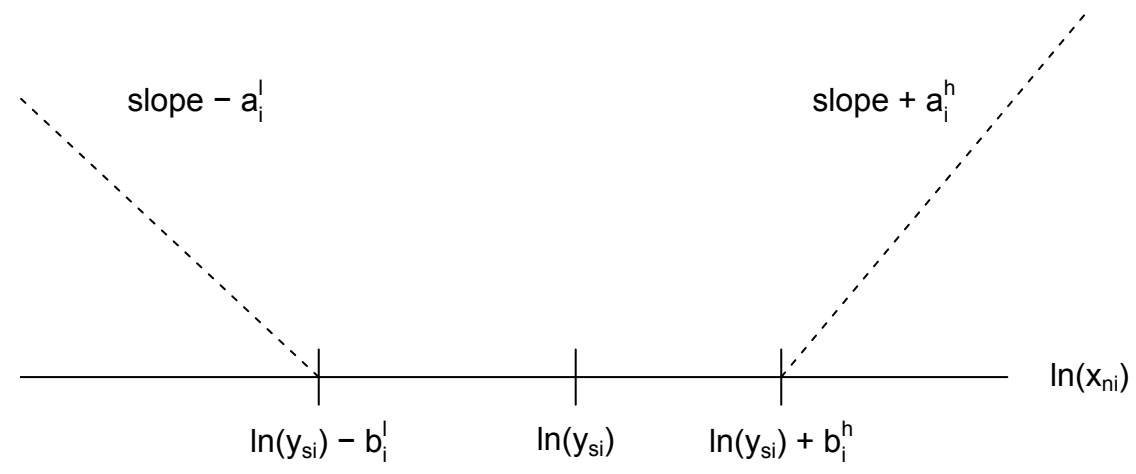

Figure 1. This plot, based on [22], illustrates the defined dissimilarity and represents the degree of misfit between the medoids and each individual for the ith dimension.

We propose to take into account the basic ideas stated in [22] to define the distance functions. First, they argue that fit is better predicted by proportional rather than absolute differences between individual and prototype features. Second, that there is an interval where there is no difference between the values $x_{i}$ and $y_{i}$ probably because the fit is perfect although the values could be different. Third, that the distance is not symmetric (a garment wich is too small may not affect fit in the same way as one wich is too large). In particular, for a given value of $\left|x_{i}-y_{i}\right|$, the distance may be smaller if $x_{i}<y_{i}$ than if $x_{i}>y_{i}$. Finally, that dissimilarities in certain dimensions are more critical to fit than others. As McCulloch et al. [22] state, there are a wide variety of functional forms which satisfy the above requirements, but we will continue using the one that they propose, and define:

$d_{i}(x, y)= \begin{cases}a_{i}^{l}\left(\ln \left(y_{i}\right)-b_{i}^{l}-\ln \left(x_{i}\right)\right), & \text { if } \ln \left(x_{i}\right)<\ln \left(y_{i}\right)-b_{i}^{l} \\ 0, & \text { if } \ln \left(y_{i}\right)-b_{i}^{l}<\ln \left(x_{i}\right)<\ln \left(y_{i}\right)+b_{i}^{h} \\ a_{i}^{h}\left(\ln \left(x_{i}\right)-b_{i}^{h}-\ln \left(y x_{i}\right)\right), & \text { if } \ln \left(x_{i}\right)>\ln \left(y_{i}\right)+b_{i}^{h}\end{cases}$

where $a^{l}, b^{l}, a^{h}$ and $b^{h}$ are constants for each dimension. In this specification, the $b_{i}$ represents the range in which fit is judged to be perfect and the $a_{i}$ 
reflects the rate at which fit deteriorates outside this range. This distance function, illustrated in fig 1, satisfies the criteria before mentioned, and allows a great deal of flexibility through the choice of parameter values.

Once defined the dissimilarity for each feature, McCulloch et al. [22] propose to define the global dissimilarity between individuals $x$ and $y$ as a sum of squared discrepancies over each of the $p$ measurements.

$$
d(x, y)=\sum_{i=1}^{p}\left(d_{i}\left(x_{i}, y_{i}\right)\right)^{2}
$$

Although it could be more natural to consider

$$
d(x, y)=\max _{i=1} d_{i}\left(x_{i}, y_{i}\right)
$$

because we would consider the worse fit from the point of view of each feature. When the distance is defined as in eq (6), the different dissimilarities $d_{i}\left(x_{i}, y_{i}\right)$ 's are being aggregated, and in our opinion, a lot of possibilities can be opened by looking at the problem under this point of view. In particular, an Ordered Weighted Average operator can be used. An OWA operator of dimension $n$ is a mapping $f: \mathbb{R}^{n} \rightarrow \mathbb{R}$ with an associated weighting vector $W=\left(w_{1}, \ldots, w_{n}\right)$ such that $\sum_{j=1}^{n} w_{j}=1$ and where $f\left(a_{1}, \ldots, a_{n}\right)=\sum_{j=1}^{n} w_{j} b_{j}$ where $b_{j}$ is the $j$-th largest element of the collection of aggregated objects $a_{1}, \ldots, a_{n}$. In our case the values to aggregate are $a_{i}=d_{i}\left(x_{i}, y_{i}\right)$.

Appendix A: Ordered weighted averages, contains a brief introduction to OWA operators.

\section{Our data}

A sample of 10.415 Spanish females from 12 to 70 years old randomly selected from the official Postcode Address File was measured using The Vitus Smart 3D body scanner from Human Solutions, a non-intrusive laser system formed by four columns allocating the optic system, which moves from the head to the feet in ten seconds performing a sweep of the body. From the 3D mesh, 95 anthropometric measures were calculated semi-automatically combining automatic measures based on geometric characteristic points with a manual review. Women were asked to wear a standard white garment, a swimming hut, a top and a short that were designed and scaled in 5 sizes, in 
order to harmonize the measurements. The design of the garment was based on the standard ISO 20685.

In addition to physical measurements other qualitative measures were collected such as women satisfaction with their bodies. They were also asked about their size in the current Spanish sizing system. Because of the lack of consistency and rigor in the current sizing system in Spain, the answers of this question were in some cases numerical and in other qualitative: small, large, etc. and in all the cases were considered as an approximation to the real size.

Not all of the anthropometric variables are useful for establishing the sizing system. From these 95 body measurements the five most relevant features in the garment development were obtained. They were chosen for different reasons. First, we follow the recommendations of experts. Second, they are commonly used in the literature about sizing system design. Finally, they appear in the European Normative to sizing system [7]. These variable are: Bust circumference, Chest circumference, Neck to ground length, Waist circumference and Hip circumference. Taking into account the European normative, we will consider Bust circumference as the principal dimension to define the size and the other four measures as secondary dimensions. Jointly to these main features, other additional features will be used to describe each size.

Finally, a selection of 6013 women was done. Pregnant women; those who declare to be breast feeding at the time; who have undergone any type of cosmetic surgery (breast augmentation, liposuction, breast reduction, etc), and the ones younger of 20 or older than 65 ,were deleted from the data set for this study. So, our data set contained finally 5 anthropometric body measurements of 6013 spanish women. The summary statistic of these five variables can be seen in table 1 .

\begin{tabular}{|l|l|l|l|l|l|l|}
\hline Measurement $(\mathrm{cm})$ & Minimum & First Quantile & Median & Mean & Third Quantile & Maximum \\
\hline Neck to ground length & 116.4 & 132.9 & 136.8 & 137 & 140.8 & 161.9 \\
\hline Bust circumference & 73 & 87.4 & 93.3 & 95.02 & 100.7 & 145.7 \\
\hline Chest circumference & 45.91 & 90.78 & 96.37 & 97.92 & 103.7 & 150.30 \\
\hline Waist circumference & 58.60 & 75.6 & 83.10 & 84.98 & 92.40 & 167.6 \\
\hline Hip circumference & 72.8 & 98.3 & 103.3 & 104.9 & 109.9 & 170.8 \\
\hline
\end{tabular}

Table 1. Summary statistics for the five variables considered. 


\section{Results}

The data set was firstly segmented in twelve subsets (classes), taking into account bust circumference values according to the sizes defined in the European Normative to sizing system [7].The trimmed $k$-medoids algorithm (section 2.1), was applied to each segment with $k=3$ clusters, and a total of 36 sizes were obtained.

The number of random initializations was 600, with 7 steps per initialization. The proportion of trimmed sample was prefixed to $\alpha=0.01$ per segment. Regarding to the constants that define the metric (eq. 5), their values were chosen taking into account:

a As in [22], a person's feature being larger than the prototype one was penalized three times more than that being smaller $\left(b_{i}^{l}=3 b_{i}^{h}\right.$ and $a_{i}^{l}=$ $\left.3 a_{i}^{h}\right)$.

b The dissimilarity consistent with a perfect fit $\left(b_{i}^{l}\right)$ was chosen within each segment to cover all the range of values of each measurement in such a way that all the individuals would be perfectly fitted in exactly one

size, i.e. for each segment $j, b_{i}^{l}=\frac{3 \cdot \operatorname{Range}\left(\left\{x_{j_{1} i}, \ldots x_{j_{n} i}\right\}\right)}{4 k}$, where $k=3$ is the number of clusters.

c The values of $a_{i}^{h}$ were chosen, as in [22], to reflect our judgment about the relative rate at which increasing discrepancies in these measurements deteriorate fit, they are given in table 2 .

\begin{tabular}{|l|l|l|}
\hline & $a_{i}^{l}$ & $a_{i}^{h}$ \\
\hline Chest circumference & 7.5 & 22.5 \\
\hline Bust circumference & 8.3 & 25 \\
\hline Neck to ground length & 9.5 & 28.5 \\
\hline Waist circumference & 6.7 & 20 \\
\hline Hip circumference & 8.3 & 25 \\
\hline
\end{tabular}

Table 2. Constants that define the distance function in equation 5 .

On the other hand, the value of orness (see Appendix A) was 0.7. 


\subsection{Visualizing of dissimilarities}

We have summarized (and represented in fig. 2 ) the dissimilarities of our data in two dimensions by means of Classical Multidimensional Scaling. Multidimensional scaling takes a set of dissimilarities and returns a set of points such that the Euclidean distances between the points are approximately equal to their dissimilarities. We have used the function cmdscale from R ([25]). As can be seen in fig. 2, there are no separated groups, but a distribution of points covering some area of the feature space. Note that this figure summarizes a lot of information in only two dimension, and that the dissimilarity proposed in Section 2.2 is not a metric, therefore this graphic should be taken with caution, as an exploratory tool.

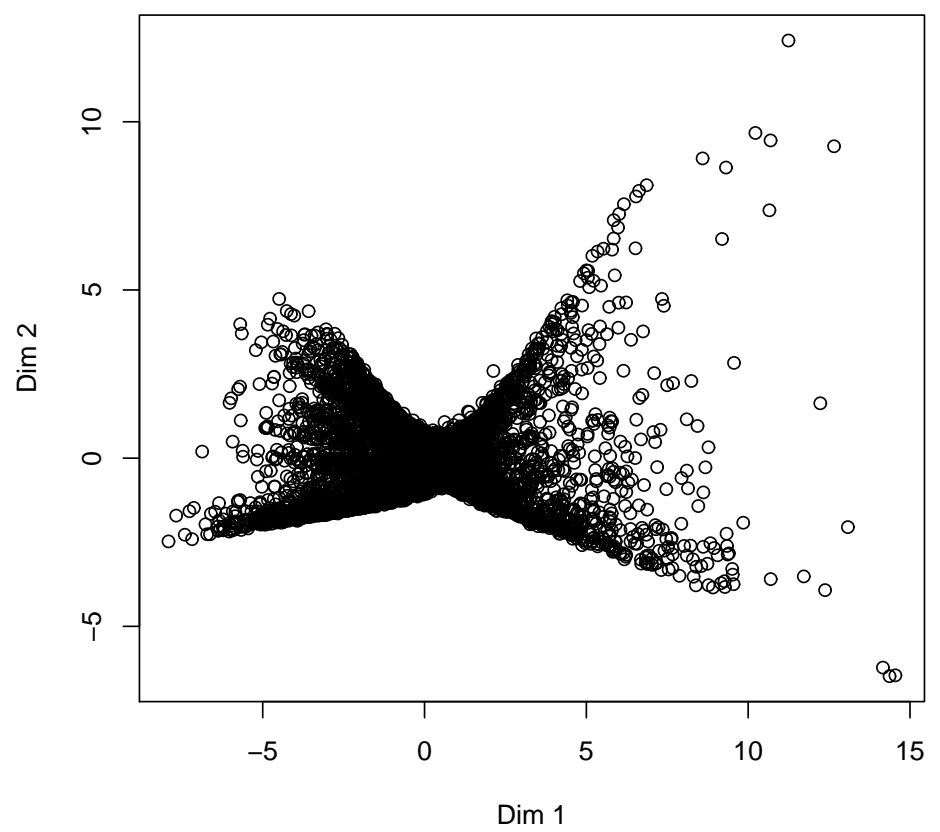

Figure 2. Two dimensional representation of woman dissimilarities (as explained in Section 2.2 ) by classical multidimensional scaling. 


\begin{tabular}{|c|c|c|c|c|c|c|c|}
\hline Woman Code & Chest & Neck to ground & Waist & Hip & Bust & Hip - Waist & Bust - Waist \\
\hline CANDE021 & 88.6283 & 132.5 & 79.1 & 99.1 & 85.3 & 20 & 6.2 \\
\hline SEVI132 & 88.6745 & 141.6 & 71.5 & 98.4 & 82.7 & 26.9 & 11.2 \\
\hline LLEID074 & 87.5182 & 135.1 & 71.1 & 96.1 & 84.5 & 20 & 13.4 \\
\hline
\end{tabular}

Table 3. Medoids measurements for bust size [82, 86[.

\begin{tabular}{|c|c|c|c|c|c|c|c|}
\hline Woman Code & Chest & Neck to ground & Waist & Hip & Bust & Hip - Waist & Bust - Waist \\
\hline SILLE034 & 96.9951 & 134.4 & 83.5 & 102.5 & 94.7 & 19 & 11.2 \\
\hline JAEN075 & 101.129 & 139.3 & 90.8 & 108.5 & 97.8 & 17.7 & 7 \\
\hline CANDE068 & 99.0432 & 139.4 & 85.3 & 104.5 & 95.7 & 19.2 & 10.4 \\
\hline
\end{tabular}

Table 4. Medoids measurements for bust size [94, 98[.

\subsection{Experimental results}

In order to illustrate our results, figs. 3 and 4 show the scatterplots of bust circumference against neck to ground (fig 3) and bust circumference against waist (fig 4), jointly with the three medoids obtained for each class. The distribution of medoids in both figures show different patterns for each bust range. As an example, lets consider the medoids obtained for women belonging to two particular bust circumference intervals: [82, 86[ and [94, 98[. Identification codes and main measurements of these medoids are detailed in tables 3 and 4 . As can be seen, medoids in range [94,98[ point out the need of only two sizes for length (medoids JAEN075 and CANDE068, have similar neck to ground measures) while medoids in range $[82,86$ [ show a greater dispersion along this variable, pointing out the adequacy of three sizes with different lengths for this bust range. The same medoids, show an opposite pattern regarding the waist measurements. For bust range [82, 86[, medoids SEVI132 and LLEID074, have similar waist circumference while the three medoids of range [94, 98 [ show quite different values for this variable. So, dissimilarity in range $[94,98$ [ is more affected by waist, while in range $[82,86[$ the variability of neck to ground predominates. 


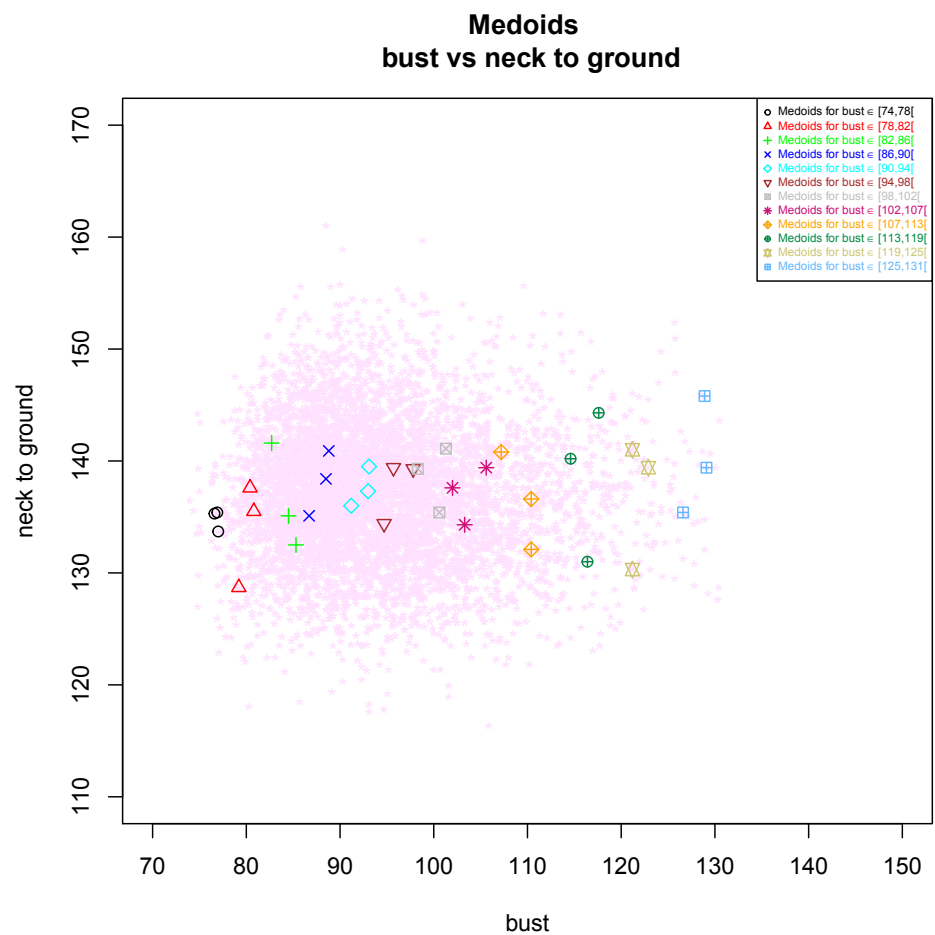

Figure 3. Bust vs neck to ground for each one of medoids. [82,86] medoids are represented with a green cross, while $[94,98]$ medoids are represented with a brown facing down triangle. 


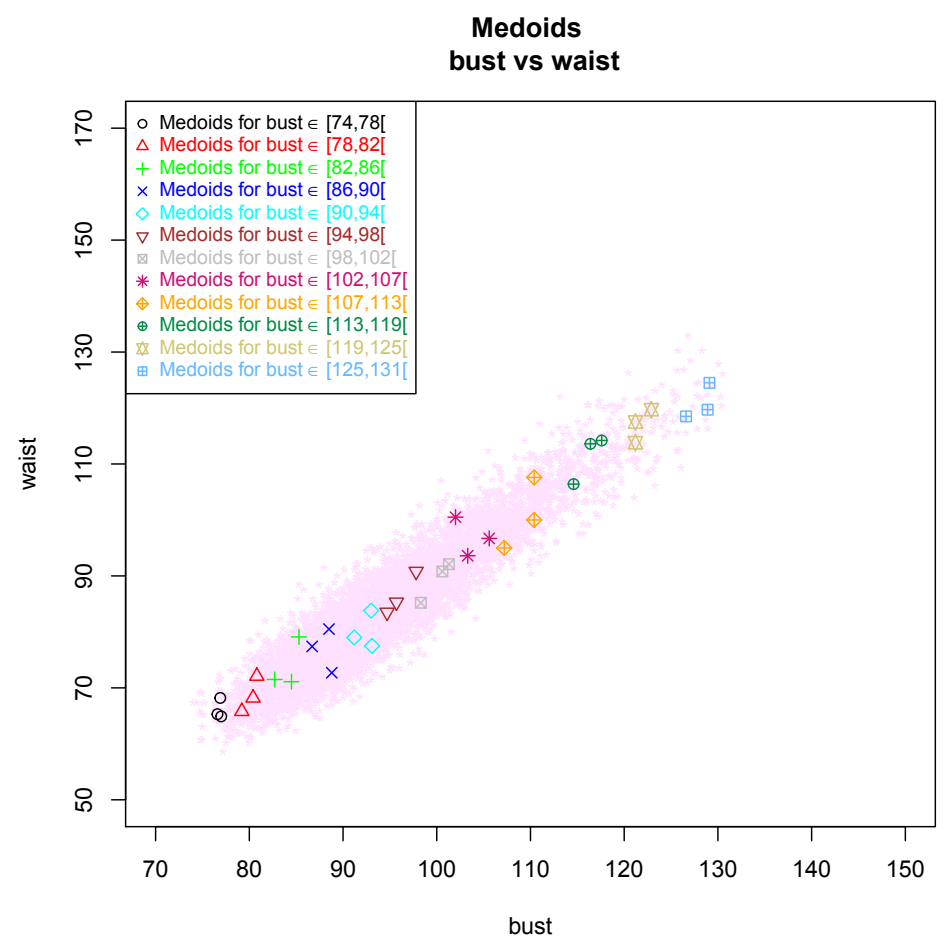

Figure 4. Bust vs waist for each one of medoids. [82,86] medoids are represented with a green cross, while [94,98] medoids are represented with a brown facing down triangle.

Figs. 5 and 6 show the body shape of the medoids obtained for the class defined by bust size $[82,86[$. As can also be seen in table 3 , medoids SEVI132 and LLEID074 have similar bust-waist proportion and similar waist circumference, while their respective heights differ. In the same way, figs. 7 and 8 show the body shape of the three medoids for the bust sizes [94, 98[. JAEN075 and CANDE068 have similar neck to ground measurement (table 4), but show a different shape in the belly area affecting the measure of the waist and therefore giving different proportions between bust and waist. 


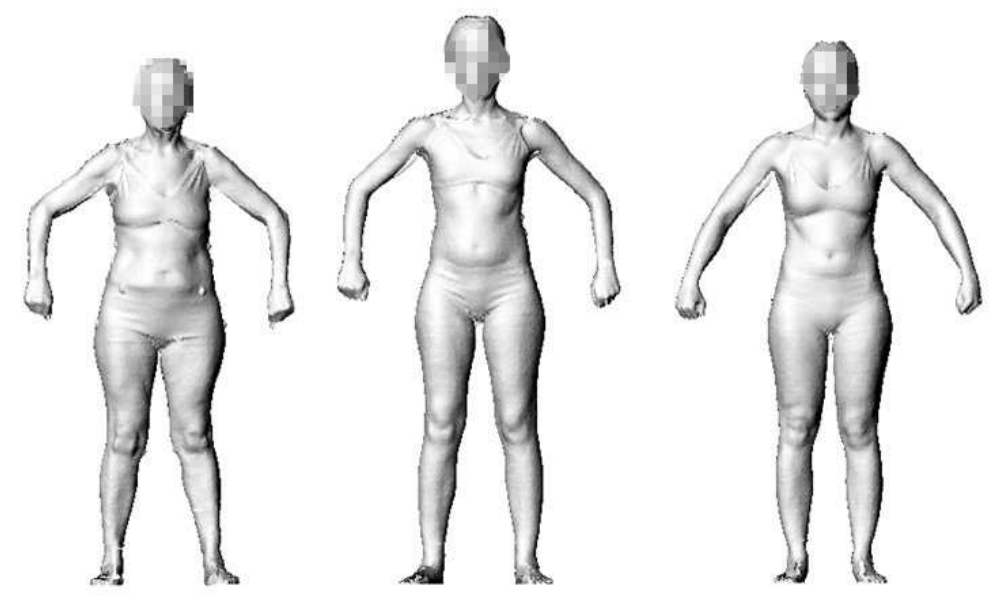

Figure 5. Front body shape of medoids for size [82,86[ (left to right, CANDE021, SEVI132 and LLEID074).
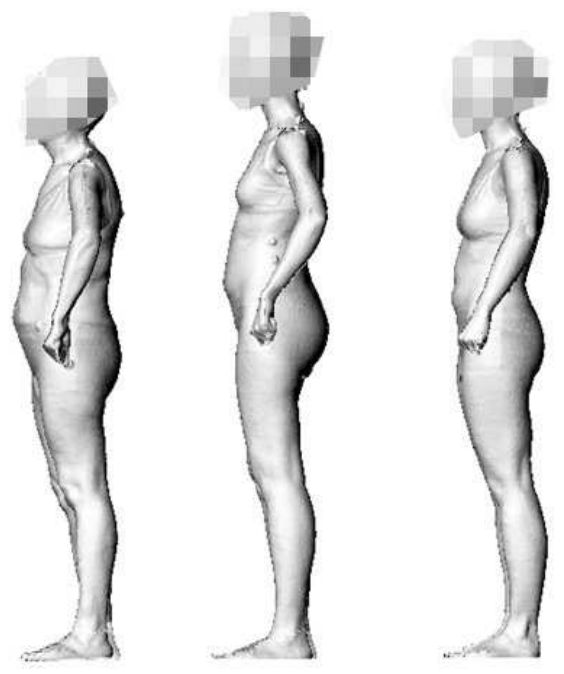

Figure 6. Lateral body shape of medoids for size [82,86[ (left to right, CANDE021, SEVI132 and LLEID074). 


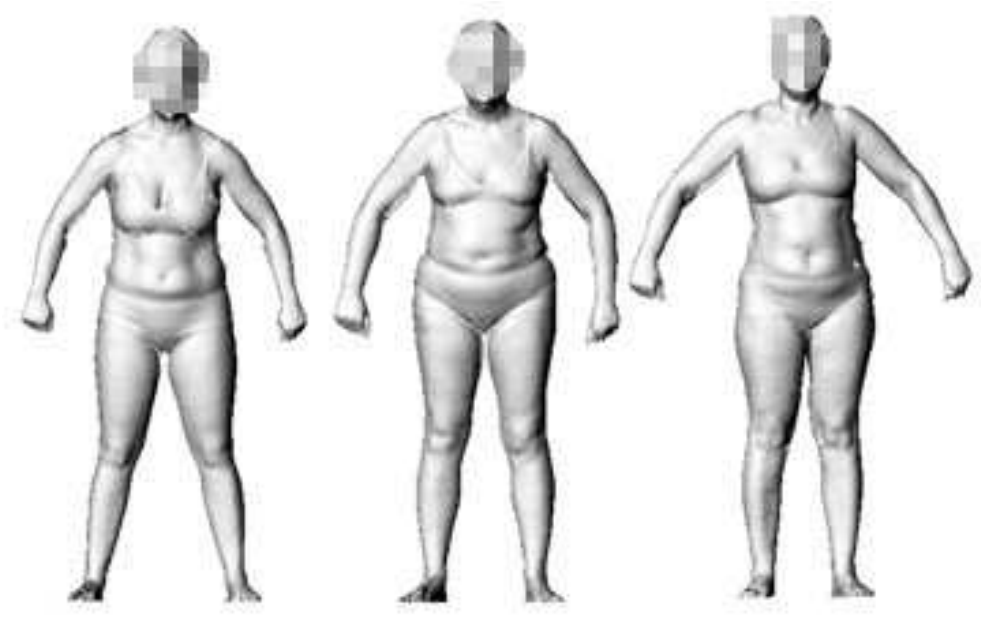

Figure 7. Front body shape of medoids for size [94,98[ (left to right, SILLE034, JAEN075 and CANDE068).
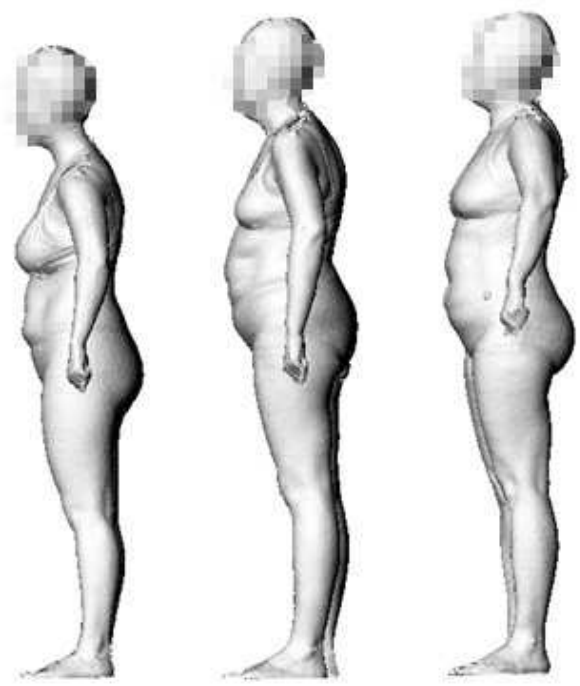

Figure 8. Lateral body shape of medoids for size [94,98[ (left to right, SILLE034, JAEN075 and CANDE068).

Finally, we would like to check the goodness of our methodology and the improvement in the garment fit, if the 36 sizes defined by our medoids were considered instead of the defined by the European Normative to sizing system 


\begin{tabular}{|c|c|c|c|c|c|c|c|c|c|c|c|c|}
\hline Bust & 76 & 80 & 84 & 88 & 92 & 96 & 100 & 104 & 116 & 122 & 128 & 134 \\
\hline Waist & 60 & 64 & 68 & 72 & 76 & 80 & 84 & 88 & 94 & 100 & 106 & 112 \\
\hline Hip & 84 & 88 & 92 & 96 & 100 & 104 & 108 & 112 & 117 & 122 & 127 & 132 \\
\hline Chest & 79.50 & 83.38 & 87.26 & 91.14 & 95.02 & 98.90 & 102.78 & 106.66 & 112.46 & 118.30 & 124.12 & 129.94 \\
\hline
\end{tabular}

Table 5. Measurement to define the sizes on the European Normative to sizing system.

[7]. This normative, establish 12 sizes according with the combinations of the bust, waist and hip measurements detailed in table 5, and does not fix chest neither height standard measurements. Anyway, given the high correlation existent between the bust and chest measurements in the women of our data set, we can approximate the chest measures through a linear regression analysis, taking the bust measures as independent variable. So, from the bust measurements detailed in the Normative, the chest measures can be approximated. The obtained values are also shown in table 5 . On the other hand, as the measurement form neck to ground shows no correlation with the other variables, we considered as neck to ground measures for the standard sizing system, the values 132, 136 and $140 \mathrm{~cm}$ because those are the most repeated measurements, and in our opinion are the measurements which best cover our data set (se fig 9). So our aim at this point is to compare the adequacy of the sizing system defined from the medoids obtained in our work, with that defined by 36 prototypes with the measurements detailed in table 5 and three different neck to ground measurements per combination. Figs 9 and 10 show two different scatterplots jointly with our medoids and the sizes obtained following the considerations of the European Normative. 


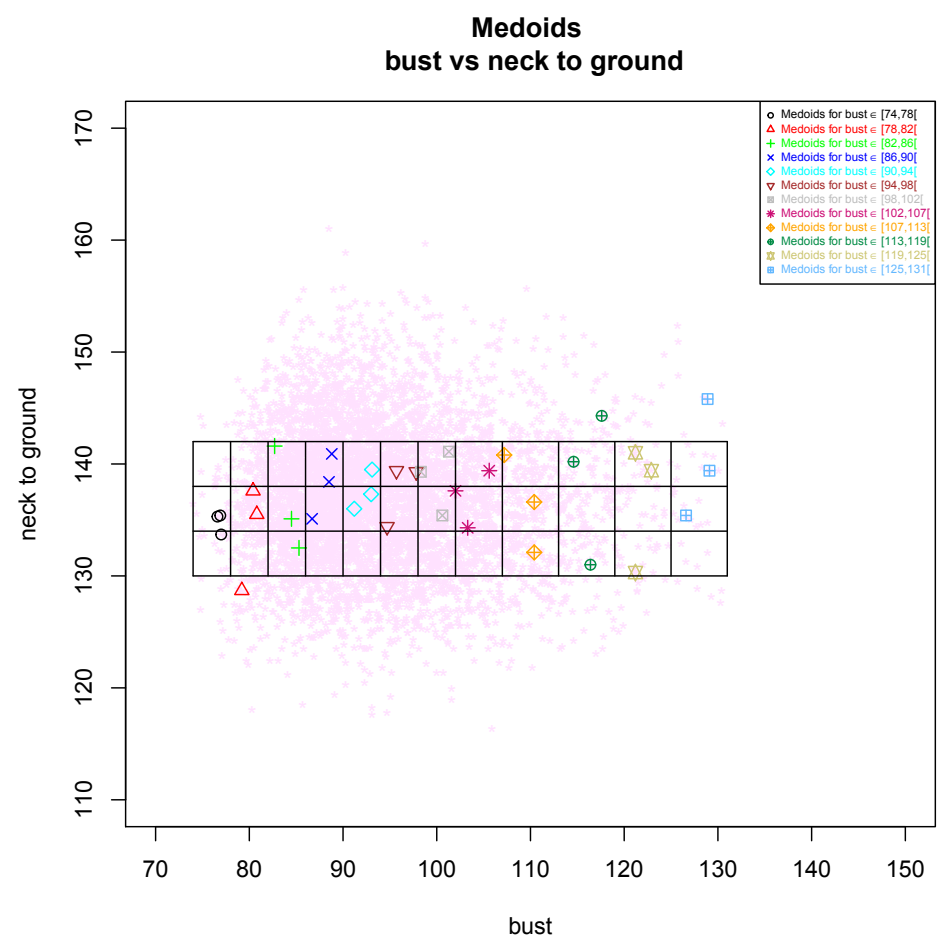

Figure 9. Bust vs Neck to ground, jointly with our medoids and the defined by the European Normative. 


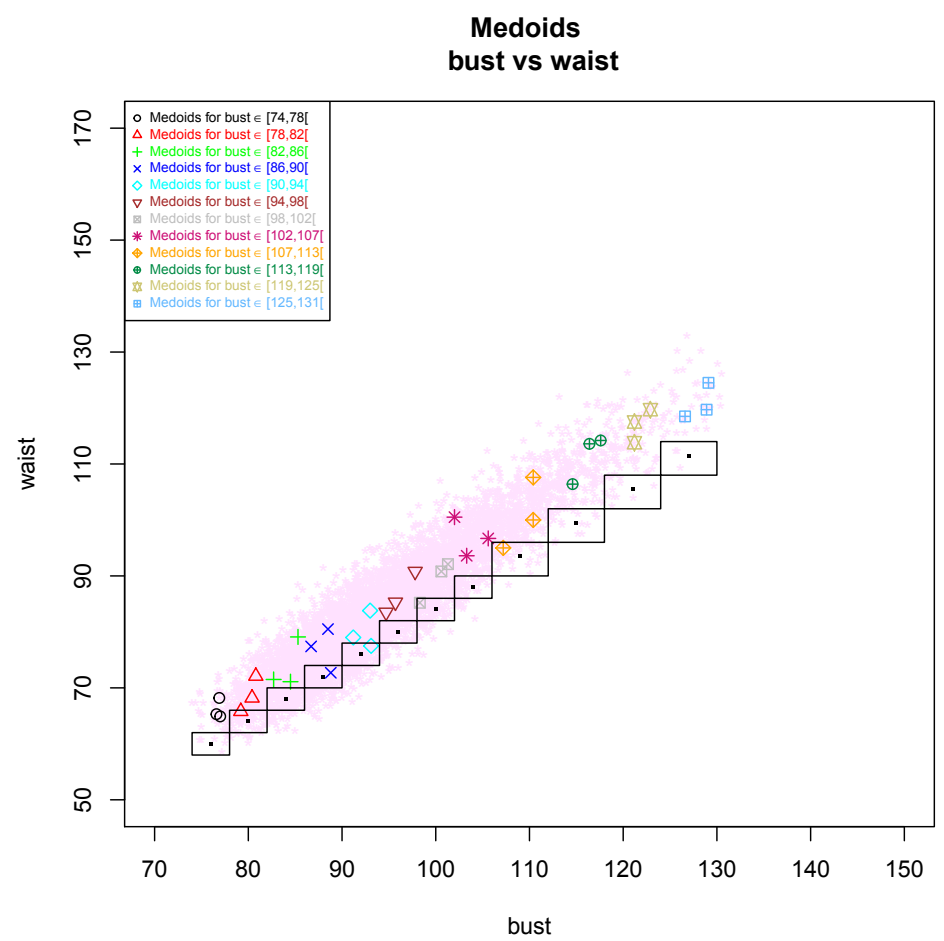

Figure 10. Bust vs waist, jointly with our medoids and the defined by the European Normative.

Finally, fig. 11 shows the cumulative distribution functions for the dissimilarities between all the women and the medoids obtained with our method and for the dissimilarities between all the women and the standard medoids defined by the European Normative to sizing system. In both cases, distances and dissimilarities have been computed by using the dissimilarity function stated in section 2.2 


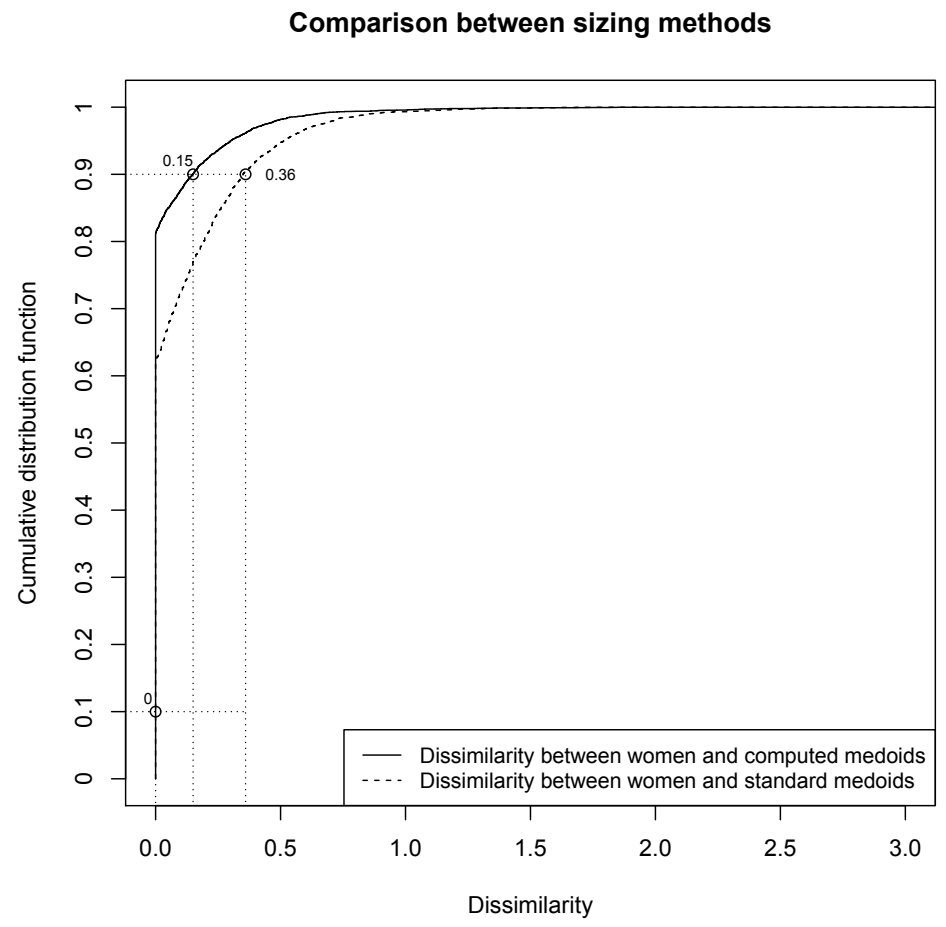

Figure 11. Cumulative distribution function for the dissimilarities between women and computed medoids and for the dissimilarities between women and standard medoids.

As we can seen in fig. 11, there is a percentage of population (rounding the $60 \%$ ), which gets a good fit in both sizing systems. With our sizing system, this percentage increases until the $80 \%$. Women measurements are closer to those of the medoids computed with our method, because the corresponding cumulative distribution function increases faster than the cumulative distribution function for the standard system.

This type of plot can also be used to identify the expected range of the dissimilarities, that is to say, the values between the 10 and 90 th percentiles. In this case, the range for the dissimilarities between women and computed medoids is $[0,0.23]$, while the range for the dissimilarities between women and standard medoids is $[0.27,3.16]$, so dissimilarities with respect to the standard medoids are greater than the dissimilarities with respect to the new computed medoids. 
For all of these reasons, this plot serves to confirm that our method build more reasonable sizes in which the women are more accommodated.

?? plots the first and second principal components of data.

\section{Conclusions}

There are two approaches in the literature to define a sizing system: traditional stepwise sizing and optimization methods. Traditional methodologies are based on segmentation of bivariant distributions of two independent variables, typically stature and waist for lower garment and stature and chest/bust for upper garment. The benefit of the traditional method is the easy way to communicate the size to consumers. On the contrary, variability of other principal anthropometric dimensions is not considered and, in consequence, a large part of the population finds not to be properly accommodated. Optimization methods try to find the minimum number of sizes that can cover the maximum percentage of population. However, the resulting sizing distribution based on multiple body dimensions presents several difficulties for a consumers find their proper size. Our study combines both approaches: the pre-segmentation based on bust, which is the primary dimension for upper garment fitting and patterning, provides a first easy input to choose the size, while the resulting morphotypes for each bust size optimize sizing using the main anthropometric dissimilarities.

A methodology to develop an apparel size system has been introduced and applied to a a recently obtained Anthropometric data base of Spanish women. The core of our approach is to segment the data set using a principal dimension (Bust circumference) and apply a trimmed- $k$-medoids algorithm with the number of sizes fixed within each class. We fix, too, a re-accommodating rate and define the discrepancy between individuals and prototypes using OWA operators. This approach has several advantages over currently used systems. Among those stated in [22], our makes simultaneously the selection of individual dis-commodities, the derivation of prototypes, and the assignment of individuals to size classes but additionally, the prototypes are more realistic because they correspond with real women of the data set. On the other hand, the use of OWA operators has resulted in a more realistic dissimilarity measure between individuals and prototypes. 


\section{References}

[1] Ashdown, S., 2007. Sizing in clothing: Developing effective sizing systems for ready-to-wear clothing. Woodhead Publishing in Textiles.

[2] Bagherzadeh, R., Latifi, M., Faramarzi, A., 2010. Employing a threestage data mining procedure to develop sizing system. World Applied Sciences Journal 8 (8), 923-929.

[3] Bye, E., LaBat, K., McKinney, E., Kim, D.-E., 2008. Optimized pattern grading. International Journal of Clothing Science and Technology $20(2), 79-92$.

[4] Chen, W., Zhuang, Z., Benson, S., Du, L., Yu, D., Landsittel, D., Wang, L., Viscusi, D., Shafer, R. E., 2009. New respirator fit test panels representing the current chinese civilian workers. The Annals of Occupational Hygiene 53 (3), 297-305.

[5] Chen, Y., Zeng, X., Happiette, M., Bruniaux, P., Ngb, R., Yu, W., 2009. Optimisation of garment design using fuzzy logic and sensory evaluation techniques. Engineering Applications of Artificial Intelligence 22, 272282.

[6] Chunga, M., Lina, H., , Wang, M.-J. J., 2007. The development of sizing systems for taiwanese elementary- and high-school students. International Journal of Industrial Ergonomics 37, 707-716.

[7] European Committee for Standardization, 2002. European Standard EN 13402-2: Size system of clothing. Primary and secondary dimensions.

[8] Fan, J., Yu, W., Hunter, L., 2004. Clothing apperance and fit: Science and technology. Woodhead Publishing in Textiles.

[9] Faust, M.-E., Carrier, S., 2010. Women's wear sizing: a new labelling system. Journal of Fashion Marketing and Management 14 (1), 88-126.

[10] García-Escudero, L. A., Gordaliza, A., Sep. 1999. Robustness properties of $\mathrm{k}$ means and trimmed k means. Journal of the American Statistical Association 94 (447), 956-969. 
[11] García-Escudero, L. A., Gordaliza, A., Matrán, C., Jun. 2003. Trimming tools in exploratory data analysis. Journal of Computational and Graphical Statistics 12 (2), 434-449.

[12] Gupta, D., Gangadhar, B., 2004. A statistical model for developing body size charts for garments. International Journal of Clothing Science and Technology 16 (5), 458-469.

[13] Hsu, C.-H., 2009. Data mining to improve industrial standards and enhance production and marketing: An empirical study in apparel industry. Expert Systems with Applications 36, 4185-4191.

[14] Hsu, C.-H., 2009. Developing accurate industrial standards to facilitate production in apparel manufacturing based on anthropometric data. Human Factors and Ergonomics in Manufacturing 19 (3), 199-211.

[15] Hsu, C.-H., Wang, M.-J. J., 2005. Using decision tree-based data mining to establish a sizing system for the manufacture of garments. The International Journal of Advanced Manufacturing Technology 26, 669-674.

[16] Iscar, A. M., Escudero, L. A. G., Fritz, H., 2010. tclust: Robust Trimmed Clustering. $\mathrm{R}$ package version 1.0-3.

URL http://CRAN.R-project.org/package=tclust

[17] Kaufman, L., Rousseeuw, P., 1990. Finding Groups in Data: An Introduction to Cluster Analysis. John Wiley, New York.

[18] La Bat, K., DeLong, M., 1990. Body cathexis and satisfaction with fit if apparel. Clothing and Textiles Research Journal 8 (2), 43-48.

[19] León, T., Zuccarello, P., Ayala, G., de Ves, E., Domingo, J., 2007. Applying logistic regression to relevance feedback in image retrieval systems. Pattern Recognition 40, 2621-2632.

[20] Luximon, A., Zhang, Y., Luximon, Y., Xiao, M., 2011. Sizing and grading for wearable products. Computer-Aided Design.

[21] Maechler, M., 2010. Cluster Analysis, extended original from P. Rousseeuw, A. Struyf and M. Hubert.

URL http://CRAN.R-project.org/package=cluster 
[22] McCulloch, C., Paal, B., Ashdown, S., 1998. An optimization approach to apparel sizing. Journal of the Operational Research Society 49, 492499.

[23] Ng, R. and Ashdown, S.P. and Chan, A., 2007. Intelligent size table generation. Proceedings of the Asian Textile Conference (ATC), 9th Asian Textile Conference, Taiwan.

[24] Pisut, G., Connell, L., 2007. Fit preferences of female consumers in the usa. Journal of Fashion Marketing and Management 11 (3), 366-379.

[25] R Development Core Team, 2009. R: A Language and Environment for Statistical Computing. R Foundation for Statistical Computing, Vienna, Austria, ISBN 3-900051-07-0.

URL http: //www.R-project.org

[26] Saaty, T., 1980. The Analytic hierarchy process: Planning, priority setting, resource allocation. McGraw-Hill.

[27] Salusso-Deonier, C., DeLong, M., Martin, F., Krohn, K., 1985-1986. A multivariate method of classifying body form variation for women's apparel. Clothing and Textiles Research Journal 4 (1), 38-45.

[28] Sizemic, 2011. http://www.sizemic.eu/products-and-services/46-size-survey-data.

[29] Smith, D. C., 2007. Process, fit and appearance analysis of threedimensional to two-dimensional automatic pattern unwrapping technology. Thesis submitted to the Graduate Faculty of North Carolina State University.

[30] Tryfos, P., 1986. An integer programming approach to the apparel sizing problem. The Journal of the Operational Research Society 37 (10), 10011006 .

[31] Yager, R., 1988. On ordered weighted averaging aggregation operators in multi-criteria decision making. IEEE Trans. Systems Man Cybernet $18,183-190$.

[32] Zheng, R., Yu, W., Fan, J., 2007. Development of a new chinese bra sizing system based on breast anthropometric measurements. International Journal of Industrial Ergonomics 37, 697-705. 


\section{Appendix A: Ordered weighted averages}

These operators were introduced in [31]. An OWA operator of dimension $n$ is a mapping $f: \mathbb{R}^{n} \rightarrow \mathbb{R}$ with an associated weighting vector $W=$ $\left(w_{1}, \ldots, w_{n}\right)$ such that $\sum_{j=1}^{n} w_{j}=1$ and where $f\left(a_{1}, \ldots, a_{n}\right)=\sum_{j=1}^{n} w_{j} b_{j}$ where $b_{j}$ is the $j$-th largest element of the collection of aggregated objects $a_{1}, \ldots, a_{n}$. The particular cases shown in table 6 can better illustrate the idea underlying OWA operators.

Table 6. Illustrating examples of OWA aggregation values.

\begin{tabular}{c|c}
$W$ & $f\left(a_{1}, \ldots, a_{n}\right)$ \\
\hline$(1,0, \ldots, 0)$ & $\max _{i} a_{i}$ \\
$(0,0, \ldots, 1)$ & $\min _{i} a_{i}$ \\
$\left(\frac{1}{n}, \frac{1}{n}, \ldots, \frac{1}{n}\right)$ & $\frac{1}{n} \sum_{j=i}^{n} a_{i}$.
\end{tabular}

As OWA operators are bounded by the max and min operators, Yager [31] introduced a quantity called orness to measure the degree to which the aggregation is like an or $(\max )$ operation:

$$
\operatorname{orness}(W)=\frac{1}{n-1} \sum_{i=1}^{n}(n-i) w_{i} \text {. }
$$

We have used a simple procedure to generate the set of weights $W=$ $\left(w_{1}, \ldots, w_{n}\right)$. They are obtained as a mixture of the binomial $\operatorname{Bi}(n-1, p)$ and the discrete uniform probability distributions, that is to say, $w_{i}=\lambda$. $\pi_{i}+(1-\lambda) \cdot \frac{1}{n}$, where $\pi_{i}$ is the binomial probability for each $i=0, \ldots, n-1$, see [19].

Remarkable advantages of this choice are its flexibility and simplicity: the weights are easily obtained and are also easy to interpret. In addition, our practical experiments have shown that it works well for this case. 